\title{
Analysis of axial prestretch in the abdominal aorta with reference to post mortem interval and degree of atherosclerosis
}

\author{
Lukas Horny $^{1 *}$, Tomas Adamek², Marketa Kulvajtova ${ }^{3}$ \\ ${ }^{1}$ Faculty of Mechanical Engineering, Czech Technical University in Prague, Technicka 4, 166 07, \\ Prague, Czech Republic \\ ${ }^{2}$ Third Faculty of Medicine, Charles University in Prague, Ruska 87, 100 00, Prague, Czech Republic \\ ${ }^{3}$ Department of Forensic Medicine, University Hospital Na Kralovskych Vinohradech, Srobarova 50, \\ 100 34, Prague, Czech Republic
}

"Corresponding author email: lukas.horny@ fs.cvut.cz, tel.: +420 224352690; fax.: +420 233322482

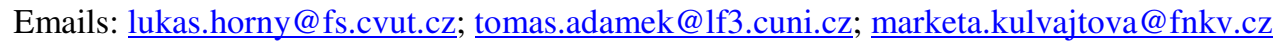

\begin{abstract}
It is a well-known fact that the length of an artery in situ and the length of an excised artery differs. Retraction of blood vessels is usually observed. This prestretch plays an important role in arterial physiology. We have recently determined that the decrease of axial prestretch in the human abdominal aorta is so closely correlated with age that it is suitable for forensic applications (estimation of the age at time of death for cadavers of unknown identity). Since post mortem autolysis may affect the reliability of an estimate based on axial prestretch, the present study aims to detail analysis of the effect of post mortem time. The abdominal aorta is a prominent site of atherosclerotic changes (ATH), which may potentially affect longitudinal prestretch. Thus ATH was also involved in the analysis. Axial prestretch in the human abdominal aorta, post mortem interval (PMI), and the degree of ATH were documented in 365 regular autopsies. The data was first age adjusted to remove any supposed correlation with age. After the age adjustment of the sample, the correlation analysis showed no significant PMI effects on the prestretch in non-putrefied bodies. Analysis of the prestretch variance with respect to ATH suggested that ATH is not a suitable factor to explain the prestretch variability remaining after the age adjustment. It was concluded that, although atherosclerotic plaques may certainly change the biomechanics of arteries, they do not significantly affect the longitudinal prestretch in the human abdominal aorta.
\end{abstract}

Keywords: aorta; age estimation; atherosclerosis; forensic biomechanics; post mortem changes 
Horny L, Adamek T, Kulvajtova M (2014) Analysis of axial prestretch in the abdominal aorta with reference to post mortem interval and degree of atherosclerosis. Journal of the Mechanical Behavior of Biomedical Materials, 33:93-98. DOI: 10.1016/j.jmbbm.2013.01.03 MANUSCRIPT VERSION

\section{INTRODUCTION}

Elastic arteries (aorta; carotid, iliac and femoral arteries) in situ are significantly prestretched in the axial direction (Horny et al., 2011; Learoyd and Taylor, 1966). This phenomenon, although wellknown, has rarely studied in detail, and human data, in particular, can only be found in a limited number of reports. Nevertheless, axial prestretch (and corresponding prestress) in an artery has an important physiological function. In an idealised case, it enables the artery to carry the pressure pulse with minimal variation in its length (Dobrin and Doyle, 1970; Schulze-Bauer and Holzapfel, 2003; Sommer et al., 2010).

However, it was found that ageing leads to significant changes in the magnitude of axial prestretch. This prestretch decrease follows the age so closely that it can be utilised as a simple measure estimating age at the time of death (Horny et al., 2012), which is one of the first steps in forensic practice when cadavers with unknown identity are autopsied. There are several methods used to this end: radiological examination of teeth and skeleton, methods of analytical chemistry like aspartic acid racemisation rate, or currently developed methods of molecular biology dealing with ageing induced DNA damage and shortening of telomeres (Cunha et al., 2009; Meissner and RitzTimme, 2010; Buk et al., 2012; Ritz-Timme et al., 2000; Dobberstein et al., 2010).

In previous reports it has been shown that age estimation based on axial prestretch (especially when a diameter-to-prestretch ratio is used) gives the same or better uncertainty in comparison with a radiological examination (Horny et al., 2012a). An advantage of the method, however, lies especially in its simplicity and accessibility. There is no delay between an autopsy and its processing, no sample preparation, no extra device is necessary; the estimate is in fact determined in the autopsy room.

It was a step from cardiovascular biomechanics towards forensic tissue biomechanics and we emphasised reporting anthropological data (prestretch, diameter, and atherosclerosis). A discussion of autopsy conditions (e.g. post mortem interval, PMI) was not performed in detail. Nevertheless, the effect of PMI is of crucial importance in both biomechanics and forensic practice. In forensic practice, PMI is probably the simplest measure indicating the onset and/or progress of post mortal changes (but of course, it is only a phenomenological, not a causal, factor). Such an analysis, however, necessitates data to be adjusted with respect to age to eliminate the possibility that a decreased/increased prestretch is observed due to an age change. However, age adjustment significantly increases the number of observations required to achieve reliable results. At present our sample has reached the number of 365 observations, which is sufficient for this purpose.

Since axial prestretch in the abdominal aorta has been suggested as an age predictor for autopsied cadavers, the results have been communicated in the form of a regression equation plus an uncertainty quantified by $95 \%$-prediction interval (PI). It was found, when diameter-to-prestretch ratio is used as the predictor, that the corresponding width of $95 \%$-PI is \pm 12.5 years (Horny et al., 2012a). Conversely, considering a man aged, e.g. 40 years, it can be shown that the expected prestretch is 1.16 with $95 \%$ - 
Horny L, Adamek T, Kulvajtova M (2014) Analysis of axial prestretch in the abdominal aorta with reference to post mortem interval and degree of atherosclerosis. Journal of the Mechanical Behavior of Biomedical Materials, 33:93-98. DOI: 10.1016/j.jmbbm.2013.01.03 MANUSCRIPT VERSION

PI equal to [1.08; 1.26] (Horny et al., 2011). Although, as mentioned above, these values are comparable with, e.g. radiological examination, it is not clear what a source of this variability is. Naturally, PMI may play a role, but individual healthy conditions may also contribute. It is well known that the abdominal aorta is a prominent site of atherosclerotic disease (ATH). Calcification and deposition of fatty substances in ATH plaques change the biomechanics of the arterial wall. Due to this fact, ATH will also be involved in the analysis. Since ATH positively correlates with age, its involvement also necessitates data to be age adjusted.

The present analysis was designed to show that axial prestretch in the human abdominal aorta measured during autopsies, reported by our team, is not significantly biased by PMI. It will also be shown that the degree of atherosclerosis does not seem to be a factor capable of explaining the remaining variability after the age adjustment.

\section{METHODS}

Data describing the in situ and excised lengths of the human abdominal aorta, as well as the age, degree of atherosclerosis (ATH) and PMI were collected during regular autopsies of Caucasian cadavers of a known age and time of the death. The post-mortem usage of human tissue was approved by the Ethics Committee of the University Hospital Královské Vinohrady in Prague. No putrefied bodies were involved. Furthermore, tortuous and aneurysmatic aortas were not involved due to a loss of straightness of their vessel axis. The degree of atherosclerosis was examined by experienced pathologist and quantified in a scale from 0 to 4 according to morphological features: 0 - intact artery and fatty streaks; 1 - fibro-fatty plaques; 2 - advanced plaques; 3 - calcified plaques; 4 - ruptured plaques (Kumar et al., 2010). It has been proven that the magnitude of axial prestretch in the abdominal aorta is not gender-specific, thus male and female data was pooled together (Horny et al., 2012a).

\subsection{Axial prestretch}

The abdominal aorta was thoroughly removed and the distance between two markers in situ and after the excision was measured with a ruler. Markers were made just below the renal arteries and above the aortoiliac bifurcation. Axial prestretch was quantified by means of the stretch ratio $\lambda$ (1). Here $l$ denotes in situ length and $L$ is the length after removal from the body.

\subsection{Age adjustment}

$$
\lambda=\frac{l}{L}
$$

In previous studies, we have reported the axial prestretch to not be closely correlated with PMI (Horny et al., 2011, 2012a). This statement was derived from an analysis handling the whole data sample (not age-adjusted). But, the prestretch exhibited noticeable variance. It seems to be natural to hypothesise 
Horny L, Adamek T, Kulvajtova M (2014) Analysis of axial prestretch in the abdominal aorta with reference to post mortem interval and degree of atherosclerosis. Journal of the Mechanical Behavior of Biomedical Materials, 33:93-98. DOI: 10.1016/j.jmbbm.2013.01.03 MANUSCRIPT VERSION

that the source of the variability comes from varying post mortem times (this is of course an unavoidable fact in autopsy measurements, where vis major play a role, in contrast to controlled conditions when animal models are employed).

In order to eliminate the age-dependent variance, the data sample was divided into intervals with non-significant effects of age. Since previous analysis showed the power law $y=a x^{b}$ to be more successful than simple linear regression, at first logarithms of the data (Age; $\lambda$ ) were calculated. It allows the classical linear regression model to be used. It was postulated that the intervals will create subsets where the model of constant $(\ln \lambda=\ln a)$ is more successful than linear regression equation $(\ln \lambda$ $=\ln a+b \cdot \ln \mathrm{Age}$ ). To this end a standard $t$-test of the hypothesis $\mathrm{H}_{0}: b=0$ (against $\mathrm{H}_{\mathrm{A}}: b \neq 0$ ) was employed. Significance level $\alpha=0.05$ was considered within this study. Final intervals were adopted as the largest intervals supporting $\mathrm{H}_{0}$ with the property that subsequent appending of at least three consecutive observations lead to the rejection of the hypothesis $\mathrm{H}_{0}: b=0$. This approach ensured that the end-points of intervals correspond to the situation where age-related trend starts to be significant. After the creation of the intervals, the correlation with age was checked by computing the linear correlation coefficient $R$ (2). The Shapiro-Wilk test was used to check the normality of data in the intervals.

$$
R=\frac{\sum_{i=1}^{n}\left(x_{i}-\bar{x}\right)\left(y_{i}-\bar{y}\right)}{\sqrt{\sum_{i=1}^{n}\left(x_{i}-\bar{x}\right)^{2} \sum_{i=1}^{n}\left(y_{i}-\bar{y}\right)^{2}}}
$$

\subsection{PMI effect and atherosclerosis}

Correlation analysis was performed to reveal mutual dependence between PMI and $\lambda$ in each age interval. It is supplemented with the test of the hypothesis $\mathrm{H}_{0}: R=0(t$-test; $\alpha=0.05)$.

The infrarenal aorta is a prominent site of atherosclerosis (Zarins et al., 2001). Previous studies have shown the degree of atherosclerosis (quantified ATH $=0, . .4$ ) correlates with $\lambda$. However, this may be a consequence of the close correlation between ATH and age. To elucidate whether ATH is responsible for the variability in $\lambda$, the analysis of variance (ANOVA) between subgroups of the prestretch classified with respect to ATH was performed in each age interval. Only subgroups with at least four elements were considered. The Bartlett test was used to confirm equal variances in subgroups. In case of non-homogenous variances, the Welch test was used instead of ANOVA. Post hoc analysis employed the Tukey test to identify which subgroups differ. The significance level $\alpha=$ 0.05 was considered. 
Horny L, Adamek T, Kulvajtova M (2014) Analysis of axial prestretch in the abdominal aorta with reference to post mortem interval and degree of atherosclerosis. Journal of the Mechanical Behavior of Biomedical Materials, 33:93-98. DOI: 10.1016/j.jmbbm.2013.01.03 MANUSCRIPT VERSION

\section{RESULTS}

Approximately two years of data collection resulted in 365 donors. The overall characteristic of the sample is as follows (mean/SSD; sample standard deviation): age - 50/17; $\lambda-1.14 / 0.10$; PMI - 47/30; and ATH - 2/4 (median/mode).

The adjustment procedure identified seven age intervals (see Table 1). Statistical tests confirmed non-significant correlation with age within these intervals $\left(\mathrm{H}_{0}: R=0\right.$ accepted). The situation is depicted in Figure 1. The normality of $\lambda$ in each interval was also confirmed.

Results of the correlation analysis for PMI and $\lambda$ are in Table 1. Low values of $R$ were found $(R \leq$ 0.2 ) and statistical evidences against $\mathrm{H}_{0}: R=0$ (i.e. no correlation between PMI and $\lambda$ ) do not exist. Data are graphically presented in Figure 2. For the sake of convenience, mean PMI and hypothetical regression lines are included (but we note that this is just the line corresponding to the alternative, not accepted, hypothesis, hence regression equations are omitted). It was concluded that the post mortem interval does not significantly affect the axial prestretch measured in autopsy when PMI $<47 / 30 \mathrm{hrs}$ (mean/SSD). In other words, it means that at a given age the variability in $\lambda$ comes from a factor other than the PMI in which it was obtained.

To reveal whether the observed variability originates in atherosclerotic disease, data in each interval were sorted into groups according to ATH. The results of ANOVA (and the Welch test in case of non-homogenous variances) are presented in Table 1. It was found, contradictory to our a priori expectation, that there are intervals were the hypothesis of systematic effect given by atherosclerosis should be rejected. In only two situations (29-34 yrs.; and 55.5-66 yrs.) ATH sorts $\lambda$ into significantly different groups. However in the interval 55.5-66 yrs. the Tukey test did not identify specific pairs which mutually differ. Detailed comparison between prestretches classified with respect to ATH is depicted in Figure 3. The figure shows the ambiguous effect of ATH on $\lambda$. Although age intervals 4655.5-66 yrs. give the impression of the decreased mean prestretch with an increased level of ATH, intervals 34-46 yrs and 66-87 yrs do not confirm such trend. The interval 29-34 yrs. even suggests an opposite trend. Under this situation, it was concluded that the degree of atherosclerosis cannot unambiguously explain the variability observed in $\lambda$.

\section{DISCUSSION}

Present studies supplement previous reports of age-related changes in longitudinal prestretch in the human abdominal aorta (Horny et al., 2011, 2012) with detailed analysis of the influence of PMI and ATH. It was found that PMI in our sample had no significant effect on the observed $\lambda$. With regard to ATH, the data did not give unambiguous results.

Mechanical experiments with elastolytically treated arteries and tissue samples obtained from genetically modified animals (with a defect in elastin synthesis) have shown medial elastin membranes to be crucial for developing and carrying longitudinal prestretch (Carta et al., 2009; Dobrin et al., 
Horny L, Adamek T, Kulvajtova M (2014) Analysis of axial prestretch in the abdominal aorta with reference to post mortem interval and degree of atherosclerosis. Journal of the Mechanical Behavior of Biomedical Materials, 33:93-98. DOI: 10.1016/j.jmbbm.2013.01.03 MANUSCRIPT VERSION

1990; Lee et al., 2012; Wagenseil and Mecham, 2012). Atherosclerosis, although a focal disease related to intima (inner layer of the artery wall; Persy and D'Haese, 2009), can affect arterial elastin. During plaque formation, cells (macrophages, monocytes, and smooth muscle cells) infiltrate a lesion. These cells release elastolytic enzymes which damage the internal elastic lamina and other adjacent elastin membranes (depending on advancement of atherosclerotic plaque; Corti and Fuster, 2003; Pyle and Young, 2010; Orlandi et al., 2006; van der Wal and Becker, 1999). Moreover, atherosclerotic plaques generally change the mechanical behaviour of the wall. These facts motivated us to involve ATH as a variable potentially correlated with the prestretch. A negative result indicates atherosclerotic plaques in the abdominal aorta do not damage a large enough number of elastic membranes to induce a substantial loss of longitudinal prestretch. This conclusion is valid, however, only for abdominal aorta with more than 25 elastic membranes (in humans; Wolinsky and Glagov, 1969). Arteries (e.g. coronary arteries) with a substantially smaller number of elastic membranes might show different results. Although our result shows ATH is not a process underlying the loss of prestretch in the human abdominal aorta, it is a key finding from the forensic point of view, which in other words means that the prestretch method is suitable for forensic practice (no further correction for ATH is necessary). It is worth noting that individuals older than 60 years without atherosclerosis are really exceptional (see Table 1).

Since ATH was not successful in capturing the remaining variability of the prestretch after age adjustment, other factors should be considered. Damage to elastin membranes, frequently described in literature as a fragmentation and disruption (Fritze et al., 2012), may come from an age-induced imbalance between proteosynthetic and proteolytic activity (Greenwald, 2007). Chemically impaired elastin fibres may subsequently be more susceptible to fatigue damage due to repetitive pressure cycles. Medial elastocalcinosis (sometimes referred to as arteriosclerosis or Monckeberg sclerosis) is also well-known for its impact on the biomechanics of elastic arteries (Dao et al., 2005; Greenwald, 2007). These factors accompany arterial ageing. However, they initiate (and progress) depending on individual conditions which may induce the variability observed in the prestretch measurement. Further studies are needed to elucidate how much they contribute to the loss of longitudinal prestretch.

The present study confirmed that $\lambda$ is not significantly changed during PMI up to 47 hours. Forty seven hours is the mean value in our sample and is used as a measure of position. However, the data in Figure 2 shows that including observations up to approximately 100 hours of PMI does not deviate PMI $-\lambda$ dependence to any significant trend (there is no significant correlation). Our study did not involve cadavers with any obvious signs of putrefaction which could explain the post mortem duration of elastic tissue properties and thus non-significant changes in the longitudinal prestretch during PMI. We should note that the onset of post mortal changes depends on the temperature. Thus studies conducted under different climate conditions may reveal somewhat different PMI effects. 
Horny L, Adamek T, Kulvajtova M (2014) Analysis of axial prestretch in the abdominal aorta with reference to post mortem interval and degree of atherosclerosis. Journal of the Mechanical Behavior of Biomedical Materials, 33:93-98. DOI: 10.1016/j.jmbbm.2013.01.03 MANUSCRIPT VERSION

\section{ACKNOWLEDGEMENT}

This work has been supported by the Czech Technical University in Prague under project SGS10/247/OHK2/3T/12, Czech Ministry of Health project NT 13302, and by Technology agency of the Czech Republic in the project TA 01010185.

\section{REFERENCES}

Buk, Z., Kordik, P., Bruzek, J., Schmitt, A., Snorek, M., 2012. The age at death assessment in a multiethnic sample of pelvic bones using nature-inspired data mining methods. Forensic Sci. Int. 220, e1e9. doi: 10.1016/j.forsciint.2012.02.019

Carta, L., Wagenseil, J.E., Knutsen, R.H., Mariko, B., Faury, G., Davis, E.C., et al., 2009. Discrete contributions of elastic fiber components to arterial development and mechanical compliance. Arterioscler. Thromb. Vasc. Biol. 29, 2083-2089. doi: 10.1161/ATVBAHA.109.193227

Corti, R., Fuster, V., 2003. New understanding, diagnosis, and prognosis of atherothrombosis and the role of imaging. Am. J. Cardiol. 91, 17A-26A. doi: 10.1016/S0002-9149(02)03146-6

CunHa, E., Baccino, E., Martrille, L., Ramsthaler, F., Prieto, J., Schuliar, Y., et al., 2009. The problem of aging human remains and living individuals: A review. Forensic Sci Int. 193, 1-13. doi: 10.1016/j.forsciint.2009.09.008

Dao, H.H., Essalihi, R., Bouvet, C., Moreau, P., 2005. Evolution and modulation of age-related medial elastocalcinosis: Impact on large artery stiffness and isolated systolic hypertension. Cardiovasc. Res. 66, 307-317. doi: 10.1016/j.cardiores.2005.01.012

Dobberstein, R.C., Tung, S.-M., Ritz-Timme, S., 2010. Aspartic acid racemisation in purified elastin from arteries as basis for age estimation. Int. J. Leg. Med. 124, 269-275. doi: 10.1007/s00414-0090392-1

Dobrin, P.B., Doyle, J.M., 1970. Vascular smooth muscle and the anisotropy of dog carotid artery. Circ. Res. 27, 105-119. doi: 10.1161/01.RES.27.1.105

Dobrin, P.B., Schwarcz, T.H., Mirkvicka, R., 1990. Longitudinal retractive force in pressurized dog and human arteries. J. Surg. Res. 48, 116-120. doi: 10.1016/0022-4804(90)90202-D

Greenwald, S.E., 2007. Ageing of the conduit arteries. J. Pathol. 211, 157-172. doi: 10.1002/path.2101

Fritze, O., Romero, B., Schleicher, M., Jacob, M.P., Oh, D.-Y., Starcher, B., et al., 2012. Age-related changes in the elastic tissue of the human aorta. J. Vasc. Res. 49, 77-86. doi: 10.1159/000331278

Horny, L., Adamek, T., Gultova, E., Zitny, R., Vesely, J., Chlup, H., Konvickova, S., 2011. Correlations between age, prestrain, diameter and atherosclerosis in the male abdominal aorta. J. Mech. Behav. Biomed. Mater. 4, 2128-2132. doi: 10.1016/j.jmbbm.2011.07.011

Horny, L., Adamek, T., Chlup, H., Zitny, R., 2012a. Age estimation based on a combined arteriosclerotic index. Int. J. Leg. Med. 126, 321-326. doi: 10.1007/s00414-011-0653-7

Horny, L., Adamek, T., Vesely, J., Chlup, H., Zitny, R., Konvickova, S., 2012b. Age-related distribution of longitudinal pre-strain in abdominal aorta with emphasis on forensic application. Forensic Sci. Int. 214, 18-22. doi: 10.1016/j.forsciint.2011.07.007

Kumar, V., Abbas, A.K., Fausto, N., Aster, J.C., 2010. Robbins and Cotran Pathologic Basis of Disease, eighth ed., Elsevier Saunders, Philadelphia.

Learoyd, B.M., Taylor, M.G., 1966. Alterations with age in the viscoelastic properties of human arterial walls. Circ. Res. 18, 278-292. doi: 10.1161/01.RES.18.3.278

Lee, A.Y., Han, B., Lamm, S.D., Fierro, C.A., Han, H.-C., 2012. Effects of elastin degradation and surrounding matrix support on artery stability. Am. J. Physiol. - Heart Circ. Physiol. 302, H873H884. doi: 10.1152/ajpheart.00463.2011 
Horny L, Adamek T, Kulvajtova M (2014) Analysis of axial prestretch in the abdominal aorta with reference to post mortem interval and degree of atherosclerosis. Journal of the Mechanical Behavior of Biomedical Materials, 33:93-98. DOI: 10.1016/j.jmbbm.2013.01.03 MANUSCRIPT VERSION

Meissner, C., Ritz-Timme, S., 2010. Molecular pathology and age estimation. Forensic Sci. Int. 203, 34-43. doi: 10.1016/j.forsciint.2010.07.010

Orlandi, A., Bochaton-Piallat, M.-L., Gabbiani, G., Spagnoli, L.G., 2006. Aging, smooth muscle cells and vascular pathobiology: Implications for atherosclerosis. Atherosclerosis 188, 221-230. doi: 10.1016/j.atherosclerosis.2006.01.018

Persy, V., D'Haese, P., 2009. Vascular calcification and bone disease: the calcification paradox. Trends. Mol. Med. 15, 405-416. doi: 10.1016/j.molmed.2009.07.001

Pyle, A.L., Young, P.P., 2010. Atheromas feel the pressure: Biomechanical stress and atherosclerosis. Am. J. Pathol. 177, 4-9. doi: 10.2353/ajpath.2010.090615

Ritz-Timme, S., Cattaneo, C., Collins, M.J., Waite, E.R., Schütz, H.W., Kaatsch, H.-J., Borrman, H.I.M., 2000. Age estimation: The state of the art in relation to the specific demands of forensic practise. Int. J. Leg. Med. 113, 129-136. doi: 10.1007/s004140050283

Schulze-Bauer, C.A.J., Holzapfel, G.A., 2003. Determination of constitutive equations for human arteries from clinical data. J. Biomech., 36, 165-169. doi: 10.1016/S0021-9290(02)00367-6

Sommer, G., Regitnig, P., Költringer, L., Holzapfel, G.A., 2010. Biaxial mechanical properties of intact and layer-disected human carotid arteries at physiological and supraphysiological loadings. Am. J. Physiol. - Heart Circ. Physiol. 298, 898-912. doi: 10.1152/ajpheart.00378.2009

van der Wal, A.C., Becker, A.E., 1999. Atherosclerotic plaque rupture-pathologic basis of plaque stability and instability. Cardiovasc. Res. 41, 334-44. doi: 10.1016/S0008-6363(98)00276-4

Wagenseil, J.E., Mecham, R.P., 2012. Elastin in large artery stiffness and hypertension. J. of Cardiovasc. Trans. Res. 5, 264-273. doi: 10.1007/s12265-012-9349-8

Wolinsky, H., Glagov, S., 1969. Comparison of abdominal and thoracic aortic medial structure in mammals. Circ. Res. 25, 677-686.

Zarins, C.K., Xu, C., Glagov, S., 2001. Atherosclerotic enlargement of the human abdominal aorta. Atherosclerosis 155, 157-164. doi: 10.1016/S0021-9150(00)00527-X 
Horny L, Adamek T, Kulvajtova M (2014) Analysis of axial prestretch in the abdominal aorta with reference to post mortem interval and degree of atherosclerosis. Journal of the Mechanical Behavior of Biomedical Materials, 33:93-98. DOI: 10.1016/j.jmbbm.2013.01.03 MANUSCRIPT VERSION

Table 1 Data summary and statistical results. Total number of observation points is 365 . SSD denotes sample standard deviation; $R$ denotes linear correlation coefficient. Significance level $\alpha=0.05$ is considered (null hypothesis, $R=0$, is rejected when probability of an error is less than 0.05). ANOVA for $\lambda$ with respect to ATH degrees involved only classes with at least four observation points. In ANOVA the null hypothesis states that inter-classes differences does not exist. Thus the hypothesis is rejected when $p$-value $<0.05$. *The results with negligible differences would be obtained employing $\ln (\lambda)$ and $\ln ($ Age $)$, due to this fact they are omitted. 'Instead of ANOVA the Welch's test was used due to non-homogenous variances. NS = non significant in Tukey HSD test.

Intervals of non-significant correlation with age

\begin{tabular}{|c|c|c|c|c|c|c|c|}
\hline Age [yrs] & $15-21.5$ & 21.5-29 & 29-34 & $34-46$ & $46-55.5$ & $55.5-66$ & $66-87$ \\
\hline $\mathbf{n}$ & 12 & 38 & 39 & 54 & 71 & 90 & 61 \\
\hline$\lambda_{\text {mean }}[-]$ & 1.37 & 1.30 & 1.22 & 1.16 & 1.10 & 1.07 & 1.05 \\
\hline $\operatorname{SSD}(\lambda)$ & 0.05 & 0.06 & 0.06 & 0.05 & 0.04 & 0.03 & 0.03 \\
\hline $\begin{array}{l}R(\lambda, \text { Age })^{*} \\
p\left(\mathbf{H}_{0}: R=0\right)\end{array}$ & $\begin{array}{c}-0.46 \\
0.13\end{array}$ & $\begin{array}{c}-0.25 \\
0.13\end{array}$ & $\begin{array}{c}-0.31 \\
0.05\end{array}$ & $\begin{array}{c}-0.25 \\
0.07\end{array}$ & $\begin{array}{c}-0.23 \\
0.05\end{array}$ & $\begin{array}{l}-0.20 \\
0.06\end{array}$ & $\begin{array}{c}-0.25 \\
0.06\end{array}$ \\
\hline PMI $_{\text {mean }}[\mathrm{hrs}]$ & 28 & 40 & 46 & 48 & 51 & 46 & 51 \\
\hline SSD(PMI) & 12 & 27 & 29 & 28 & 32 & 29 & 35 \\
\hline $\begin{array}{c}R(\lambda, \mathrm{PMI}) \\
p\left(\mathrm{H}_{0}: R=0\right)\end{array}$ & $\begin{array}{c}-0.13 \\
0.68\end{array}$ & $\begin{array}{l}0.16 \\
0.32\end{array}$ & $\begin{array}{c}-0.02 \\
0.89\end{array}$ & $\begin{array}{c}-0.20 \\
0.14\end{array}$ & $\begin{array}{l}0.12 \\
0.31\end{array}$ & $\begin{array}{l}0.16 \\
0.13\end{array}$ & $\begin{array}{c}-0.03 \\
0.85\end{array}$ \\
\hline $\mathbf{A T H}_{\text {meadian }}[-]$ & 0 & 0 & 0 & 2 & 2 & 3 & 4 \\
\hline $\begin{array}{c}\mathrm{ATH}_{\text {frequency }} \\
{[\mathbf{0 , 1} \mathbf{2}, \mathbf{3 , 4}]}\end{array}$ & $12,0,0,0,0$ & $21,16,1,0,0$ & $23,11,4,1,0$ & $15,8,27,1,3$ & $6,4,27,18,16$ & $2,3,17,24,44$ & $0,0,7,8,46$ \\
\hline $\begin{array}{c}p(\text { Bartlett }) \\
\text { homogenous } \\
\text { variances }\end{array}$ & - & 0.97 & 0.58 & 0.34 & 0.01 & 0.31 & 0.56 \\
\hline $\begin{array}{c}p(\text { ANOVA }) \\
\text { for groups of } \\
\lambda \text { following } \\
\text { ATH }\end{array}$ & - & 0.80 & 0.01 & 0.56 & $0.14^{\dagger}$ & 0.04 & 0.29 \\
\hline $\begin{array}{c}\text { Tukey } \alpha=0.05 \\
\text { ATH } \\
\text { subgroups }\end{array}$ & - & - & 1 vs. 3 & - & - & NS & - \\
\hline
\end{tabular}


Figure legends

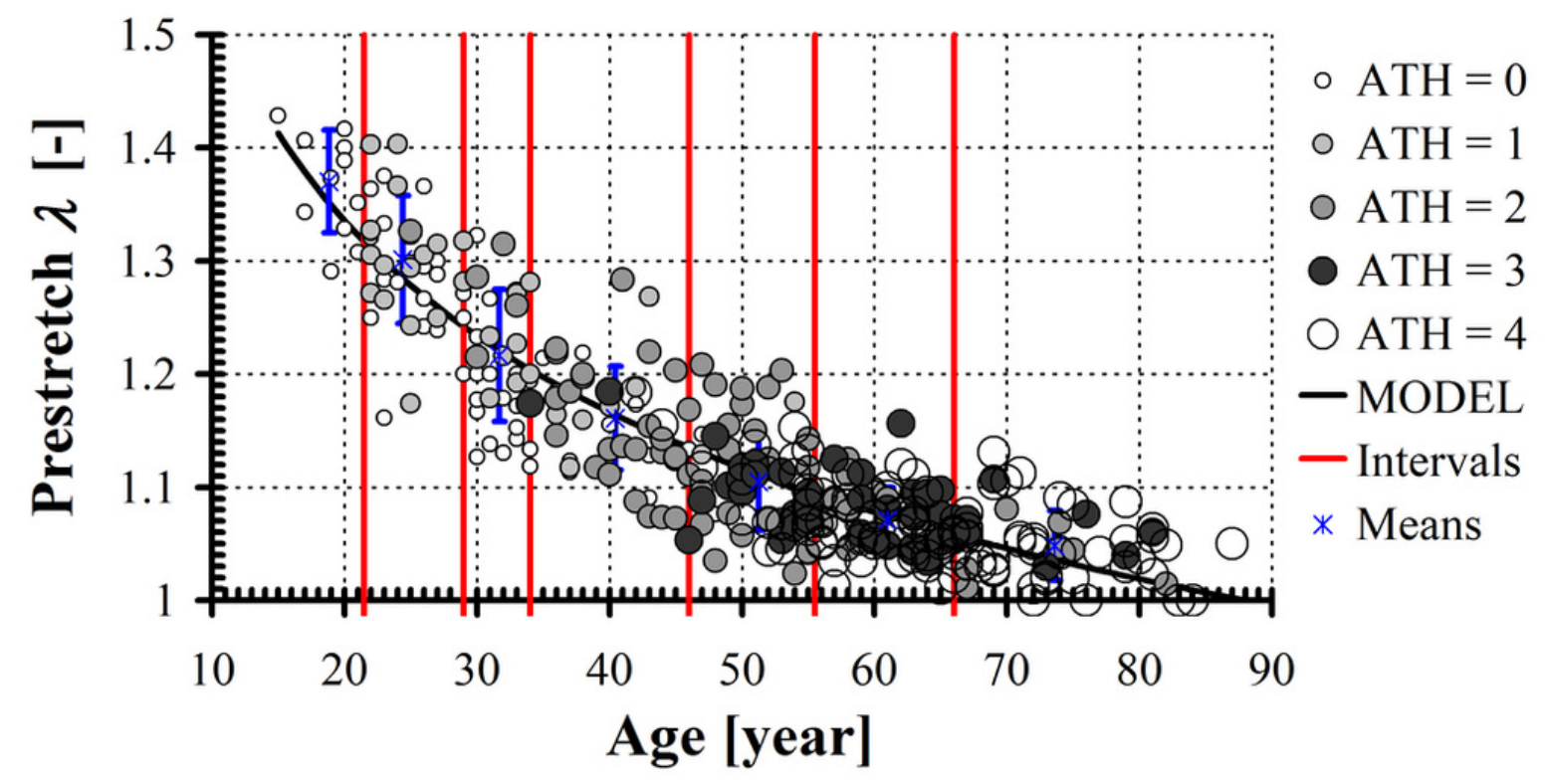

Fig. 1 Data and intervals with non-significant correlation with age. Mean value and error bars (SSD) in each interval are depicted. Data symbols indicate the degree of atherosclerosis. Least square fit gave the model $y=a x^{b}$ with parameters $a=2.3969$ [yrs] and $b=-0.1952[-] ; R(\ln (\lambda), \ln ($ Age $))=-0.90$. 
Horny L, Adamek T, Kulvajtova M (2014) Analysis of axial prestretch in the abdominal aorta with reference to post mortem interval and degree of atherosclerosis. Journal of the Mechanical Behavior of Biomedical Materials, 33:93-98. DOI: 10.1016/j.jmbbm.2013.01.03 MANUSCRIPT VERSION
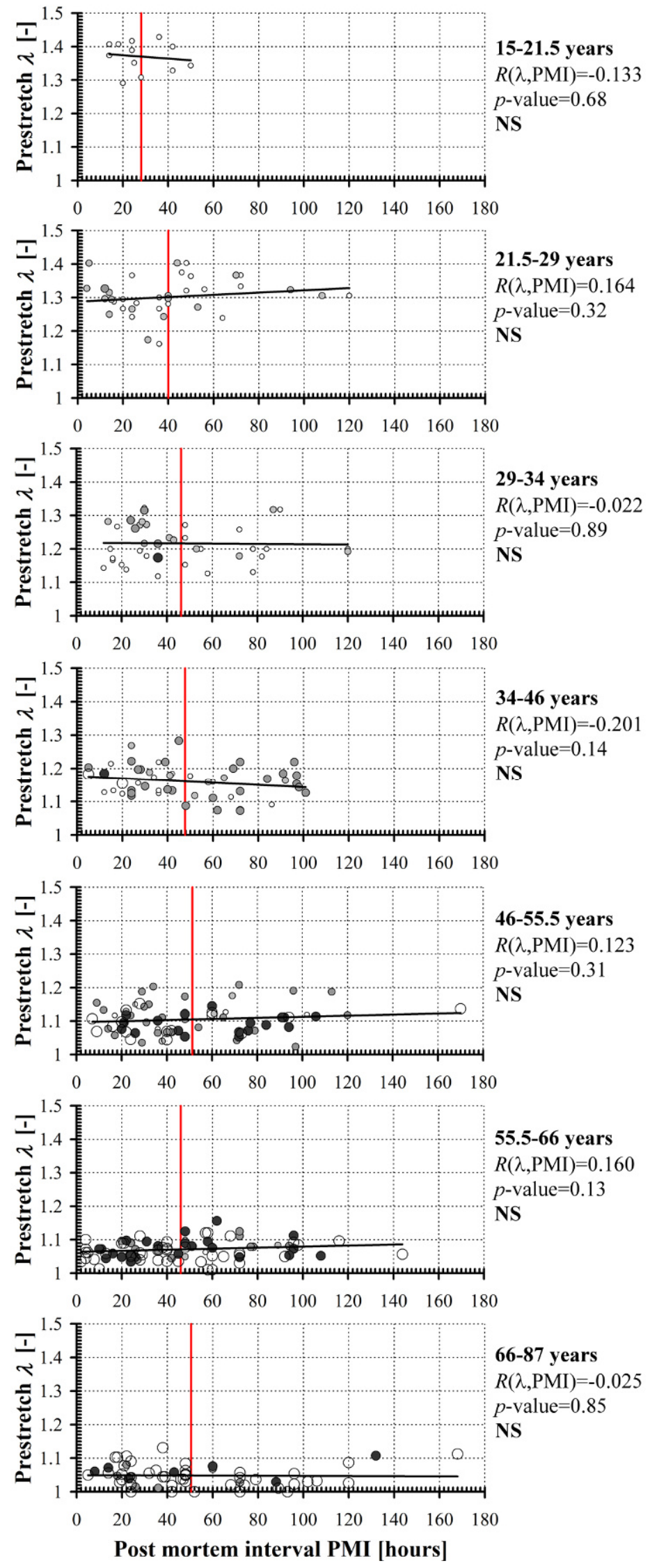

Fig. 2 Non-significant correlation with PMI in each interval was confirmed. Horizontal line shows hypothetical linear model of $\lambda$-PMI relationship. Vertical line indicates the position of mean PMI. Symbols indicate ATH in the same way as in Fig. 1. 


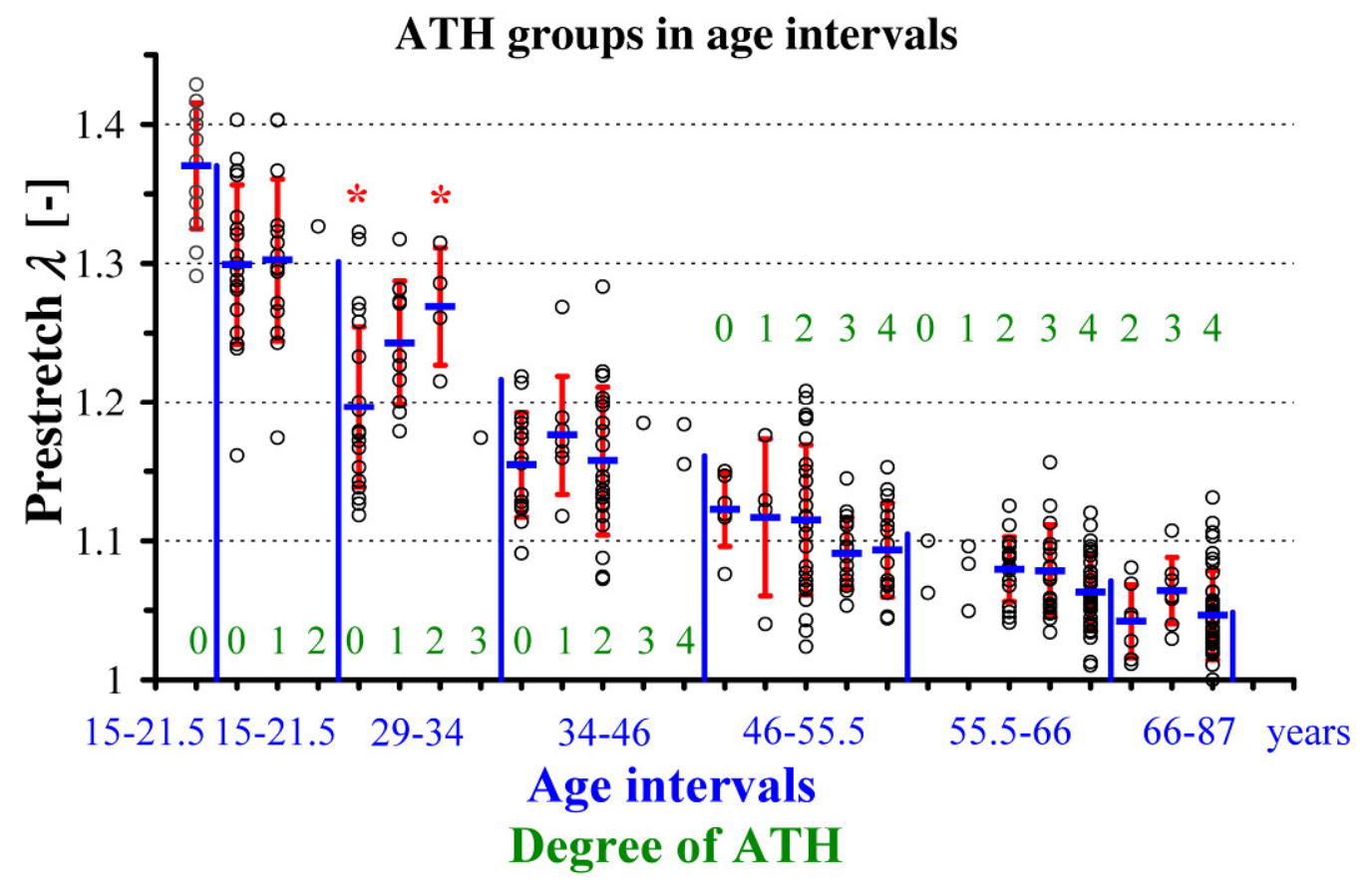

Fig. 3 Comparison of axial prestretches measured in human abdominal aorta. Age intervals are separated with vertical line (the height corresponds to average prestretch in left interval). Numerals inside the graph correspond to ATH levels. Data points in each subgroup are depicted with mean \pm SSD. Significantly different subgroups are indicated with asterisk (interval 29-34 years). Although ANOVA suggested statistical differences also in 55.5-66 years interval, Tukey test did not reveal significantly different groups. 
Horny L, Adamek T, Kulvajtova M (2014) Analysis of axial prestretch in the abdominal aorta with reference to post mortem interval and degree of atherosclerosis. Journal of the Mechanical Behavior of Biomedical Materials, 33:93-98. DOI: 10.1016/j.jmbbm.2013.01.03 MANUSCRIPT VERSION

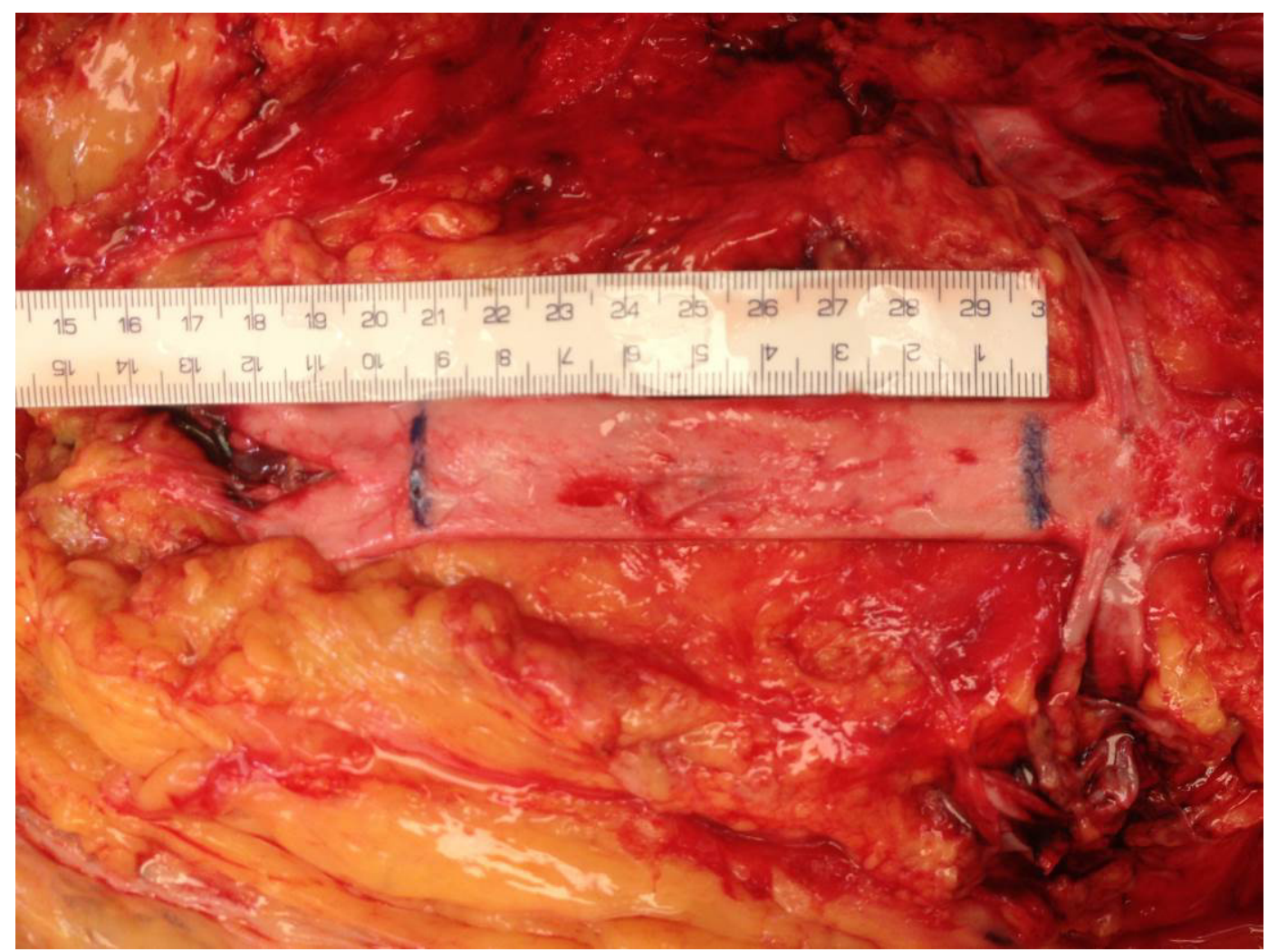

Supplement figure: Autopsied human abdominal aorta in situ

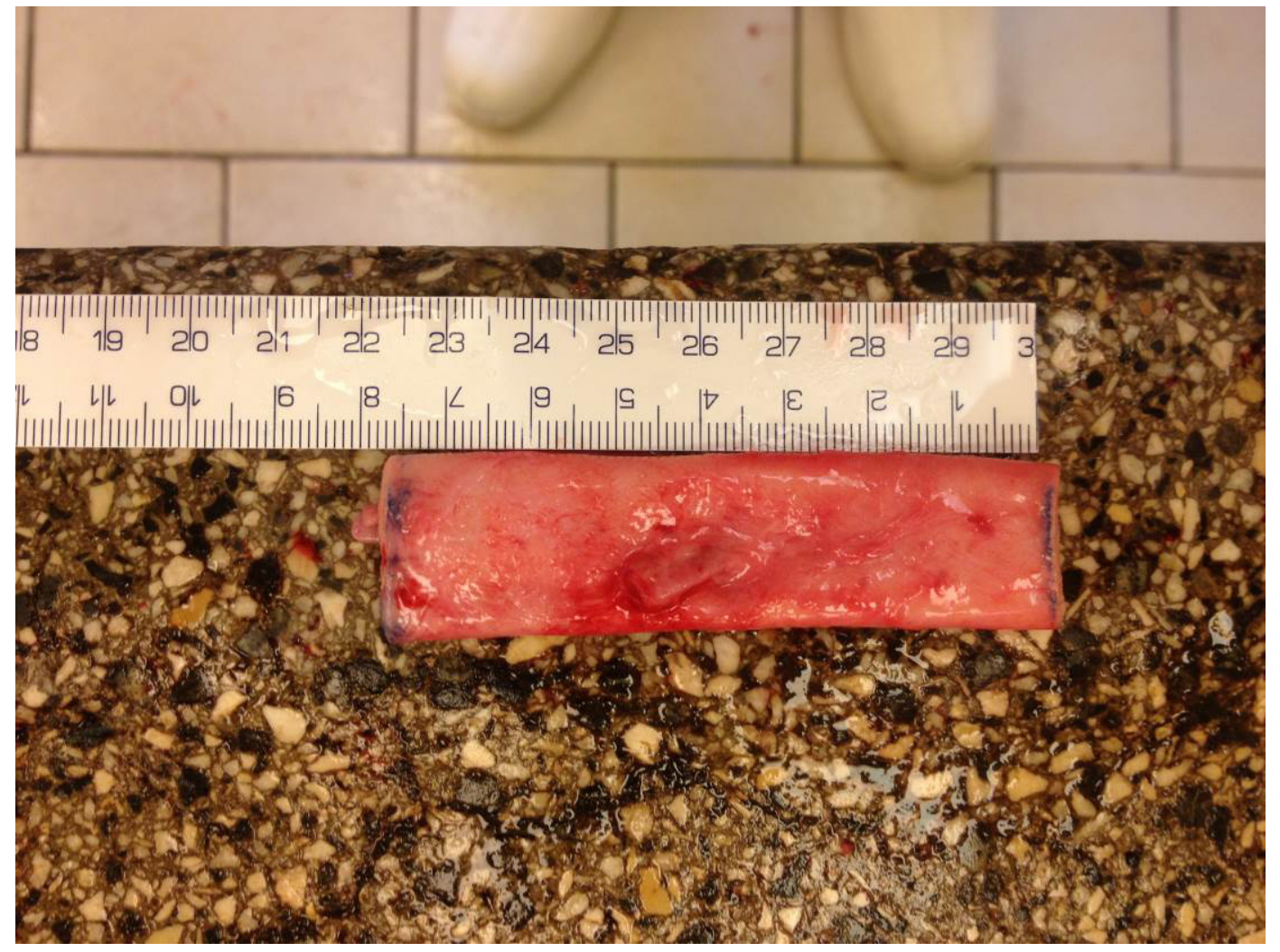

Supplement figure: Autopsied human abdominal aorta ex situ 